PROCEEDINGS OF THE

AMERICAN MATHEMATICAL SOCIETY

Volume 137, Number 1, January 2009, Pages 61-63

S 0002-9939(08)09539-7

Article electronically published on August 4, 2008

\title{
A NOTE ON THE EFFECTIVE NON-VANISHING CONJECTURE
}

\author{
QIHONG XIE
}

(Communicated by Ted Chinburg)

\begin{abstract}
We give a reduction of the irregular case for the effective nonvanishing conjecture by virtue of the Fourier-Mukai transform. As a consequence, we reprove that the effective non-vanishing conjecture holds on algebraic surfaces.
\end{abstract}

In this paper we consider the following so-called effective non-vanishing conjecture, which has been put forward by Ambro and Kawamata [Am99, Ka00].

Conjecture $1\left(E N_{n}\right)$. Let $X$ be a proper normal variety of dimension $n, B$ an effective $\mathbb{R}$-divisor on $X$ such that the pair $(X, B)$ is Kawamata log terminal, and $D$ a Cartier divisor on $X$. Assume that $D$ is nef and that $D-\left(K_{X}+B\right)$ is nef and big. Then $H^{0}(X, D) \neq 0$.

This conjecture is closely related to the minimal model program and plays an important role in the classification theory of Fano varieties. For a detailed introduction to this conjecture, we refer the reader to Xie06.

By the Kawamata-Viehweg vanishing theorem, we have $H^{i}(X, D)=0$ for any positive integer $i$. Thus $H^{0}(X, D) \neq 0$ is equivalent to $\chi(X, D) \neq 0$. Under the same assumptions as in Conjecture 1, the Kawamata-Shokurov non-vanishing theorem says that $H^{0}(X, m D) \neq 0$ for all $m \gg 0$. Thus the effective non-vanishing conjecture is an improvement of the non-vanishing theorem in some sense.

Note that $E N_{1}$ is trivial by the Riemann-Roch theorem and that $E N_{2}$ was settled by Kawamata Ka00, Theorem 3.1] by virtue of the logarithmic semipositivity theorem. For $n \geq 3$, only a few results are known. For instance, $E N_{n}$ holds trivially for toric varieties $\mathrm{Mu} 02$, $E N_{3}(X, 0)$ holds for all canonical projective minimal threefolds $X$ [Ka00, Proposition 4.1], and $E N_{3}(X, 0)$ also holds for almost all canonical projective threefolds $X$ with $-K_{X}$ nef [Xie05, Corollary 4.5].

In this paper, we shall prove that, in the irregular case, the effective nonvanishing conjecture can be reduced to lower-dimensional cases by means of the Fourier-Mukai transform. As consequences, $E N_{2}$ is reproved after Kawamata, and $E N_{n}$ holds for all varieties of maximal Albanese dimension.

Throughout this paper, we work over the complex number field $\mathbb{C}$. For the definition of the Kawamata log terminal (KLT, for short) and the other notions, we refer the reader to KMM87, KM98.

For irregular varieties, the study of the Albanese map provides enough information to understand their birational structure. Therefore, through the Albanese

Received by the editors January 17, 2007, and, in revised form, October 18, 2007, November 16, 2007, and December 27, 2007.

2000 Mathematics Subject Classification. Primary 14E30.

(C) 2008 American Mathematical Society 61 
map, we can utilize the Fourier-Mukai transform to give a reduction of the effective non-vanishing conjecture for irregular varieties. This idea was first used in [CH02]. First of all, we need the following lemma which follows easily from Mu81, Theorem $2.2]$.

Lemma 2. Let $A$ be an abelian variety, and let $\mathcal{F}$ be a coherent sheaf on $A$. Assume that $H^{i}(A, \mathcal{F} \otimes P)=0$ for all $P \in \operatorname{Pic}^{0}(A)$ and all $i$. Then $\mathcal{F}=0$.

Proof. Let $\hat{A}$ be the dual abelian variety of $A$. The assumption implies that the Fourier-Mukai transform $\Phi(\mathcal{F})$ of $\mathcal{F}$ is the zero sheaf on $\hat{A}$. Since the Fourier-Mukai transform $\Phi: D(A) \rightarrow D(\hat{A})$ induces an equivalence of derived categories Mu81, Theorem 2.2], we have $\mathcal{F}=0$.

Theorem 3. If $E N_{k}$ holds for any $k<n$, then $E N_{n}(X, B)$ holds for any $X$ with irregularity $q(X):=\operatorname{dim} H^{1}\left(X, \mathcal{O}_{X}\right)>0$.

Proof. By Kodaira's lemma, we may assume that $H=D-\left(K_{X}+B\right)$ is ample and $B$ is a $\mathbb{Q}$-divisor. Let $\pi: \widetilde{X} \rightarrow X$ be a resolution of $X$, and $\widetilde{\alpha}: \widetilde{X} \rightarrow A=\operatorname{Alb}(\widetilde{X})$ the Albanese morphism of $\widetilde{X}$. Since $(X, B)$ is KLT, $X$ has only rational singularities by [KM98, Theorem 5.22], hence $q(\widetilde{X})=q(X)>0$. Since there are no rational curves on $A$, we have a non-trivial proper morphism $\alpha: X \rightarrow A$.

Let $P \in \operatorname{Pic}^{0}(A), P^{\prime}=\alpha^{*} P$ and $\mathcal{F}=\alpha_{*} \mathcal{O}_{X}(D)$. By the Kawamata-Viehweg vanishing theorem, we have $H^{i}\left(X, D+P^{\prime}\right)=0$ for any $i>0$. By the relative Kawamata-Viehweg vanishing theorem [KMM87, Theorem 1-2-5], we have $R^{i} \alpha_{*} \mathcal{O}_{X}\left(D+P^{\prime}\right)=0$ for any $i>0$. It follows from the Leray spectral sequence that $H^{i}(A, \mathcal{F} \otimes P)=H^{i}\left(X, D+P^{\prime}\right)=0$ for any $i>0$. If $H^{0}(A, \mathcal{F})=0$, then $h^{0}(A, \mathcal{F} \otimes P)=\chi(A, \mathcal{F} \otimes P)=\chi(A, \mathcal{F})=0$; i.e. $H^{0}(A, \mathcal{F} \otimes P)=0$ for all $P \in \operatorname{Pic}^{0}(A)$. By Lemma 2, we have $\mathcal{F}=0$.

Next we prove that $\mathcal{F} \neq 0$, which implies $H^{0}(X, D)=H^{0}(A, \mathcal{F}) \neq 0$. Let $a(X)=\operatorname{dim} \alpha(X)>0$. If $a(X)=n$, then $\alpha: X \rightarrow \alpha(X)$ is generically finite, and it is easy to see that $\mathcal{F} \neq 0$. Assume that $a(X)<n$. Let $f: X \rightarrow Y$ be the Stein factorization of $\alpha, F$ a general fiber of $f$ and $\mathcal{G}=f_{*} \mathcal{O}_{X}(D)$. Then $F$ is a normal proper variety of dimension less than $n$. Note that $\left.D\right|_{F}$ is nef Cartier, $\left(F,\left.B\right|_{F}\right)$ is KLT and $\left.D\right|_{F}-\left(K_{F}+\left.B\right|_{F}\right)=\left.H\right|_{F}$ is ample. By assumption, we have $\operatorname{rank} \mathcal{G}=h^{0}\left(F,\left.D\right|_{F}\right) \neq 0$; hence $\mathcal{G} \neq 0$ as well as $\mathcal{F} \neq 0$.

Corollary 4. $E N_{2}$ holds, and $E N_{3}$ holds for any $X$ with $q(X)>0$.

Proof. For $E N_{2}$, by the Riemann-Roch theorem, one has only to deal with the case where $X$ is a ruled surface over a smooth projective curve $C$ with $q(X)=$ $g(C) \geq 2$. Since $E N_{1}$ holds, $E N_{2}$ also holds by Theorem 3. The second conclusion is obvious.

Corollary 5. $E N_{n}(X, B)$ holds for any $X$ of maximal Albanese dimension.

Proof. By assumption, $X$ is of maximal Albanese dimension; i.e. the Albanese morphism $\alpha: X \rightarrow A$ satisfies $\operatorname{dim} \alpha(X)=\operatorname{dim} X=n$. So we can repeat the same argument as in Theorem 3 to complete the proof by noting that $\alpha$ is generically finite.

Remark 6. Note that Corollary 5 has already appeared in PP03] and PP05, Theorem 5.8]. Note also that the assumption that $D$ is nef in Conjecture 1 is not needed in the proof of Corollary [5, however PP05, Lemma 5.1] proved that if 
$D-\left(K_{X}+B\right)$ is nef and big, then $D$ must be nef on the variety $X$ of maximal Albanese dimension. Furthermore, when the assumption that $D-\left(K_{X}+B\right)$ is nef and big in Conjecture 1 is replaced with the weaker assumption that $D-\left(K_{X}+B\right)$ is either nef or of non-negative Iitaka dimension, $H^{0}(X, D) \neq 0$ also holds for any $X$ of maximal Albanese dimension PP06, Theorem 6.1]. Finally, we should mention that Theorem 3 and [PP05, Theorem 5.8] used a similar idea in proof.

\section{ACKNOWLEDGMENTS}

I am deeply indebted to Professors Yujiro Kawamata and Takao Fujita for valuable advice and warm encouragement. I would like to express my gratitude to Professors Giuseppe Pareschi and Mihnea Popa for pointing out some known results, which are summarized in Remark 6. I also thank the referee for useful suggestions and comments. This work was partially supported by JSPS grant No. P05044 and by the 21st Century COE Program.

\section{REFERENCES}

[Am99] F. Ambro, Ladders on Fano varieties, Algebraic Geometry, 9. J. Math. Sci., 94 (1999), 1126-1135. MR.1703912 (2000e:14067)

[CH02] J. A. Chen, C. Hacon, Linear series of irregular varieties, Algebraic Geometry in East Asia (Kyoto, 2001), World Sci. Publ., River Edge, NJ, 2002, 143-153. MR2030451 (2005a:14010)

[Ka00] Y. Kawamata, On effective non-vanishing and base-point-freeness, Asian J. Math., 4 (2000), 173-182. MR.1802918 (2002b:14010)

[KMM87] Y. Kawamata, K. Matsuda, K. Matsuki, Introduction to the minimal model problem, Alg. Geom. Sendai 1985, Adv. Stud. Pure Math., 10 (1987), 283-360. MR946243 (89e:14015)

[KM98] J. Kollár, S. Mori, Birational Geometry of Algebraic Varieties, Cambridge Tracts in Math., vol. 134, Cambridge Univ. Press, 1998. MR1658959 (2000b:14018)

[Mu81] S. Mukai, Duality between $D(X)$ and $D(\hat{X})$ with its application to Picard sheaves, Nagoya Math. J., 81 (1981), 153-175. MR607081 (82f:14036)

[Mu02] M. Mustaţă, Vanishing theorems on toric varieties, Tôhoku Math. J., 54 (2002), 451470. MR.1916637 (2003e:14013)

[PP03] G. Pareschi, M. Popa, Regularity on abelian varieties I, J. Amer. Math. Soc., 16 (2003), 285-302. MR 1949161 (2004c:14086)

[PP05] G. Pareschi, M. Popa, M-regularity and the Fourier-Mukai transform, preprint, math.AG/0512645.

[PP06] G. Pareschi, M. Popa, GV-sheaves, Fourier-Mukai transform, and generic vanishing, preprint, math.AG/0608127.

[Xie05] Q. Xie, On pseudo-effectivity of the second Chern classes for terminal threefolds, Asian J. Math., 9 (2005), 121-132. MR2150695 (2006e:14009)

[Xie06] Q. Xie, Effective non-vanishing for algebraic surfaces in positive characteristic, J. Algebra, 305 (2006), 1111-1127. MR2266872 (2007g:14048)

Department of Mathematics, Tokyo Institute of Technology, 2-12-1 Oh-okayama, MEguro, TOKYO 152-8551, JAPAN

Current address: Graduate School of Mathematical Sciences, University of Tokyo, Komaba, Meguro, Tokyo 153-8914, Japan

E-mail address: xie_qihong@hotmail.com 